\title{
Searches for physics beyond the Standard Model at HERA
}

\author{
Antje Hüttmann for the H1 and ZEUS Collaborations \\ Deutsches Elektronen-Synchrotron DESY, Notkestraße 85, 22607 Hamburg, Germany
}

DOI: http://dx.doi.org/10.5689/UA-PROC-2010-09/54

\begin{abstract}
Recent searches for physics beyond the Standard Model are performed using $e^{ \pm} p$ data, corresponding to a total integrated luminosity of about $0.5 \mathrm{fb}^{-1}$ per experiment, collected with the $\mathrm{H} 1$ and ZEUS detectors at HERA. No evidence for any signal of new physics was found. Limits were derived on the effective quark charge radius, the effective mass scale in eeqq contact interactions, the effective Planck mass scale in models with large extra dimensions, the ratio of Yukawa coupling to mass for heavy $1^{\text {st }}$ generation leptoquarks and the Yukawa couplings as a function of mass for $2^{\text {nd }} / 3^{\text {rd }}$ generation leptoquarks and squarks in R-parity violating SUSY models. Limits on the ratio of the coupling to the compositeness scale, $f / \Lambda$, as a function of the excited fermion mass were also set. In addition, searches for new physics in exclusive final states such as events with isolated leptons and large missing transverse momentum or high- $p_{T}$ multi-lepton final states are presented.
\end{abstract}

\section{Introduction}

The HERA $e^{ \pm} p$ collider (located at DESY, Hamburg) completed its operation in the year 2007, after 15 years of successful running. Electrons/positrons with an energy of $27.5 \mathrm{GeV}$ were collided with protons of energy up to $920 \mathrm{GeV}$, resulting in a centre-of-mass energy $(\sqrt{s})$ of up to $319 \mathrm{GeV}$. The H1 and ZEUS experiments have collected a data sample of a total integrated luminosity of about $0.5 \mathrm{fb}^{-1}$ per experiment. This data sample enables the search for rare phenomena, with cross sections around or below $1 \mathrm{pb}$. In addition, it offers unique opportunities to search for physics beyond the Standard Model (SM). The neutral current (NC) $e^{ \pm} p \rightarrow e^{ \pm} X$, and charge current (CC) $e^{ \pm} p \rightarrow \nu / \bar{\nu} X$, deep inelastic $e^{ \pm} p$ scattering (DIS) interactions at high virtuality of the exchanged bosons, $Q^{2}$, at HERA are an ideal environment to study Standard Model processes as well as search for new phenomena and physics beyond the SM.

\section{Quark radius}

A finite spatial distribution of the quark charge would be evidence for quark substructure. In the classical form factor approximation, $Q^{2} \ll 1 / R_{q}^{2}$, and assuming electrons to be pointlike, 
the DIS cross section at high momentum transfer $Q^{2}$ is modified according to

$$
\frac{d \sigma}{d Q^{2}}=\frac{d \sigma^{S M}}{d Q^{2}} \cdot\left[1-\frac{R_{q}^{2}}{6} Q^{2}\right]^{2},
$$

where $R_{q}$ is the root mean square radius of the electroweak charge distribution in the quark. Since no deviations from the SM prediction for NC DIS were observed, H1 (ZEUS) set an upper limit on the quark radius of $0.65 \cdot 10^{-18} \mathrm{~m}\left(0.63 \cdot 10^{-18} \mathrm{~m}\right)$, at $95 \% \mathrm{CL}$ [1, 2]. Cross section deviations corresponding to the $95 \%$ CL upper limit for the effective radius, $R_{q}$, of the electroweak charge of the quark are compared with the ZEUS data in Figure 1 (left).

\section{General contact interaction models}

Contact interactions are an effective theory describing possible low energy effects in $e^{ \pm} p$ DIS at high $Q^{2}$ coming from physics beyond the SM at much higher energy scales, $\Lambda \gg \sqrt{s}$. Vectortype eeqq contact interactions are described by an effective Lagrangian:

$$
\mathcal{L}_{C I}=\sum_{a, b=L, R}^{q=u, d} \eta_{a b}^{q}\left(\bar{e}_{a} \gamma_{\mu} e_{a}\right)\left(\bar{q}_{b} \gamma^{\mu} q_{b}\right),
$$

where the sum runs over the electron and quark helicities ( $L$ meaning left-handed and $R$ meaning right-handed polarisation) and the quark flavour (up quarks or down quarks). The couplings $\eta_{a b}^{q}$ describe the helicity structure of the contact interaction and can be related to the effective mass scale $\Lambda$ :

$$
\eta_{a b}^{q}= \pm 4 \pi / \Lambda^{2}
$$

No significant deviation of the HERA data from the SM prediction is observed. Therefore, limits on the mass scale parameters of the models are derived. The 95\% CL lower limits on the compositeness scale $\Lambda$ obtained by the H1 (ZEUS) collaboration range from $3.7(3.8) \mathrm{TeV}$ to $7.4(8.9) \mathrm{TeV}$, depending on the CI model $[1,2]$. For many models the HERA results are unique.

\section{Large extra dimensions}

In the ADD (Arkani-Hamed, Dimopoulos, Dvali) model [3], the space-time is $4+n$ dimensional. The SM particles are confined to four dimensions, while gravity can propagate into the extra dimensions. The fundamental Planck scale in $4+n$ dimensions, $M_{D}$, can be of the order of $1 \mathrm{TeV}$. In this case the strength of gravitational and electroweak interactions would be comparable at high energies and the hierarchy problem would be solved. A virtual graviton exchange contribution to $e q \rightarrow e q$ scattering can be described by a contact interaction with effective coupling strength of $\eta_{G} \sim \pm \lambda / M_{S}^{4}$, where $M_{S}$ is an ultraviolet cutoff scale, expected to be of the order of the Planck scale $M_{D}$, and the coupling $\lambda$ is of the order of unity. Since the sign of $\lambda$ is not known a priori, both values are considered: $\lambda= \pm 1$. For $\lambda=+1(\lambda=-1) \mathrm{H} 1$ set a lower limit on $M_{S}$ of $0.90 \mathrm{TeV}(0.91 \mathrm{TeV})$ at $95 \% \mathrm{CL}$ [1]. ZEUS set a lower limit on $M_{S}$ of $0.94 \mathrm{TeV}$ for both signs of $\lambda$, at $95 \% \mathrm{CL}$ [2]. In Figure 1 (right) the effects of graviton exchange on the $Q^{2}$ distribution of NC DIS events, corresponding to the ZEUS limits, are compared with the ZEUS $e^{+} p$ data. 
ZEUS

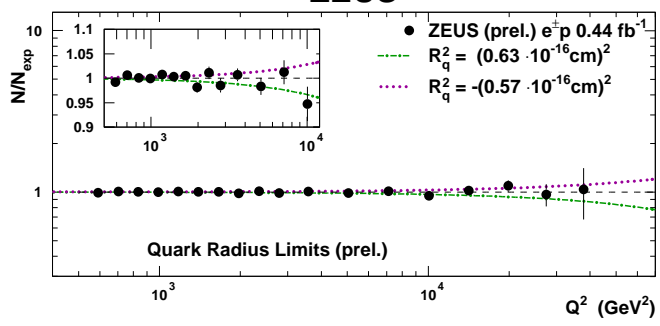

ZEUS

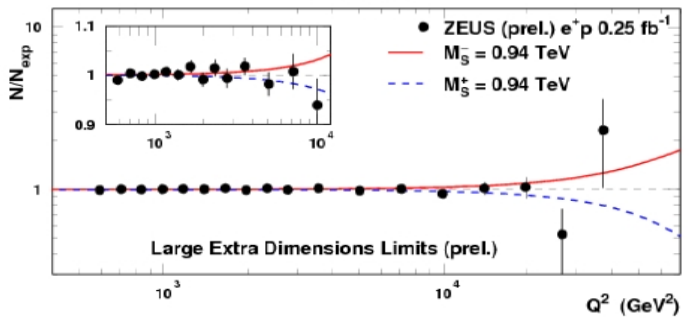

Figure 1: ZEUS $e^{ \pm} p$ data normalised to the SM expectation compared to the $95 \%$ CL exclusion limits on the quark radius (left), and the ZEUS $e^{+} p$ data normalised to the SM expectation compared to the 95\% CL exclusion limits on the effective Planck mass scale in models with large extra dimensions, for positive $\left(M_{S}^{+}\right)$and negative $\left(M_{S}^{-}\right)$couplings (right).

\section{$5 \quad$ Heavy leptoquarks}

Leptoquarks (LQ) are scalar or vector bosons carrying both lepton and baryon number, colour charge and fractional electric charge. In the framework of the Buchmüller-Rückl-Wyler model [4], which assumes flavour diagonal couplings, there are 14 different first generation leptoquark types. Heavy leptoquark exchange $\left(M_{L Q} \gg \sqrt{s}\right)$ can be described by a four-fermion contact interaction with effective coupling $\eta \sim \lambda^{2} / M_{L Q}^{2}$, where $\lambda$ is the leptoquark Yukawa coupling. Lower limits on $M_{L Q} / \lambda$ at $95 \%$ CL were set by $\mathrm{H} 1$ (ZEUS) ranging from 0.4 (0.41) TeV to $1.94(1.88) \mathrm{TeV}[1,2]$, depending on the LQ type. Figure 2 (left) shows the $\mathrm{H} 1 e^{+} p$ and $e^{-} p$ NC DIS cross section $d \sigma / d Q^{2}$ normalised to the SM expectation and compared to the exclusion limits for $S_{1}^{L}$ and $V_{1}^{L}$ leptoquarks.

\section{Lepton flavour violation}

In an extension of the Buchmüller-Rückl-Wyler model [4], the leptoquark couplings are not assumed as flavour diagonal and LQs can mediate the lepton flavour violating processes $e p \rightarrow$ $\mu X$ and $e p \rightarrow \tau X$. No evidence for lepton flavour violation is found in the full H1 data and thus limits were set on the Yukawa coupling constants $\lambda$ as a function of the leptoquark mass assuming $\lambda_{e q}=\lambda_{\mu q}$ and $\lambda_{e q}=\lambda_{\tau q}$ for $2^{\text {nd }}$ and $3^{\text {rd }}$ generation, respectively. Leptoquarks produced in $e^{ \pm} p$ collisions with a coupling strength of $\lambda=0.3$ and decaying with the same coupling strength to a $\mu q$ or $\tau q$ pair are excluded at 95\% CL up to masses of $530 \mathrm{GeV}$ and $450 \mathrm{GeV}$, respectively [5]. Figure 2 (right) shows the limits for several $2^{\text {nd }}$ generation scalar leptoquarks.

\section{$7 \quad$ Squark production in $R$-parity violating supersymmetry}

In SUSY models with $R$-parity violation single squarks can be produced resonantly in $e^{ \pm} p$ collisions. The squarks decay either to lepton and quark giving DIS-like final states or to states involving a gaugino, leading to cascade decays. No deviations from the SM were observed in 

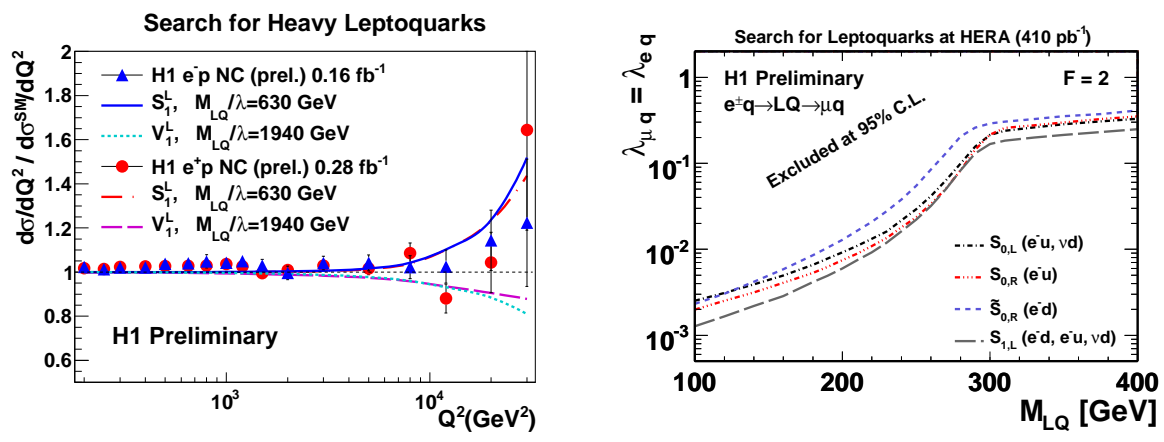

Figure 2: $\mathrm{H} 1 e^{+} p$ and $e^{-} p$ NC DIS cross section $d \sigma / d Q^{2}$ normalised to the SM expectation and compared with the $95 \%$ CL exclusion limits for the ratio of the leptoquark mass to the Yukawa coupling, $M / \lambda$, for the $S_{1}^{L}$ and $V_{1}^{L}$ leptoquarks (left), and the exclusion limit at 95\% CL on the leptoquark Yukawa coupling as a function of the leptoquark mass for scalar leptoquarks with fermion number $F=2$, assuming $\lambda_{e q}=\lambda_{\mu q}$ and $\lambda_{\tau q}=0$ (right).

any of the 17 relevant final state topologies. The H1 collaboration set mass dependent limits on the Yukawa coupling $\lambda^{\prime}$ within a phenomenological version of the MSSM [6]. The limit for $\tilde{t}_{L}$ squarks is shown in Figure 3 (left). Assuming $\lambda^{\prime}$ of the electromagnetic strength, $\tilde{u}_{L}, \tilde{c}_{L}$, $\tilde{t}_{L}\left(\tilde{d}_{R}, \tilde{s}_{R}, \tilde{b}_{R}\right)$ squarks with masses up to $\sim 275 \mathrm{GeV}(\sim 290 \mathrm{GeV})$ are excluded at 95\% CL. Furthermore, limits in the minimal Supergravity (mSUGRA) model were set in the $m_{0}-m_{1 / 2}$ plane [6], where $m_{0}\left(m_{1 / 2}\right)$ is the universal scalar (gaugino) mass at the GUT scale. Figure 3 (right) shows the limits at 95\% CL for up-type squarks assuming $\lambda_{1 j 1}^{\prime}$ of the electromagnetic strength and $\tan \beta=6$ for $j=1,2$ and 3 . Constraints obtained by the D0 experiment at Tevatron [7] are also shown in the plot.

\section{Excited fermions}

The observation of excited fermions would be direct evidence for compositeness (i.e. fermion substructure). Compositeness could explain the three lepton and the three quark families as well as their mass hierarchy. A search for excited electrons, neutrinos and quarks was performed by the H1 collaboration. Excited fermions would decay to standard fermions and gauge bosons, therefore subsequent leptonic and hadronic decay channels of $Z$ and $W$ bosons were investigated. No evidence for excited fermion production was found and thus limits were set on the ratio of the coupling to the compositeness scale, $f / \Lambda$, at $95 \% \mathrm{CL}$ as a function of the mass of the excited fermion. Assuming $f / \Lambda=1 / M_{f^{*}}$, excited quarks (electrons, neutrinos) with masses up to $252 \mathrm{GeV}(272 \mathrm{GeV}, 213 \mathrm{GeV})$ are excluded at $95 \% \mathrm{CL}$ [10].

\section{Exclusive final states}

The clean experimental signature of isolated leptons in events with missing transverse momentum and of multi-leptons with high transverse momenta, $p_{T}$, together with the precisely calculable small SM cross section provides high sensitivity to possible contributions of physics 

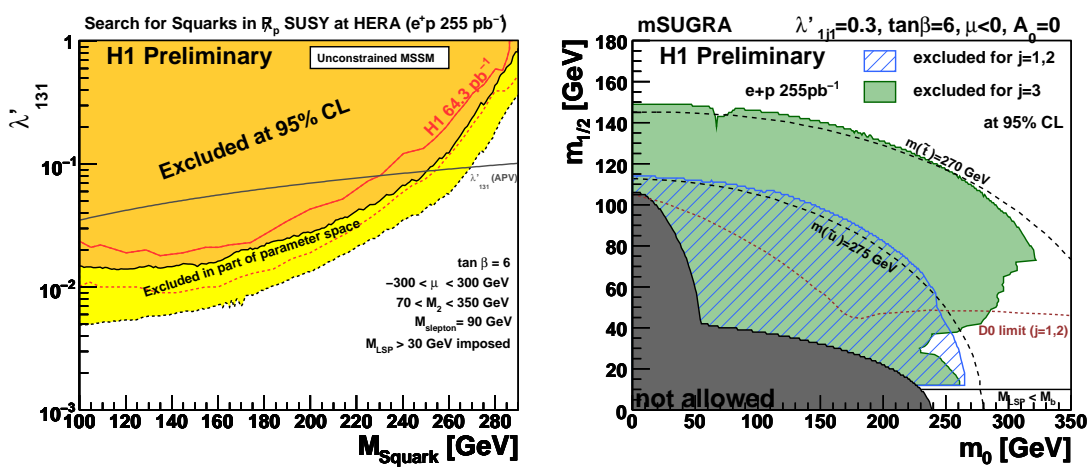

Figure 3: Exclusion limit at 95\% CL on the Yukawa coupling $\lambda_{131}^{\prime}$ as a function of the $\tilde{t}_{L}$ squark mass from a scan of the MSSM parameter space as indicated in the figure. Values of $\lambda^{\prime}$ higher than the solid curve could be excluded in all scenarios, values in between the solid and the dashed curve could be excluded in part of the parameter space investigated. Indirect limits from atomic parity violation (APV) [8] are also shown. For comparison, the corresponding limit from the HERAI analysis [9] is also presented (left). Regions excluded at 95\% CL in the $m_{0}-m_{1 / 2}$ plane for up-type squarks in the mSUGRA model assuming $\lambda_{1 j 1}^{\prime}=0.3$ and $\tan \beta=6$ for $j=1,2$ and 3 (right).

beyond the SM. The dominating SM process with isolated leptons and missing transverse momentum in the final state is single-W production. Using the combined H1 and ZEUS data [11] with an integrated luminosity of $0.98 \mathrm{fb}^{-1}, 23$ events at high transverse momentum of the hadronic system, $p_{T}^{X}>25 \mathrm{GeV}$, were observed in the $e^{+} p$ data, compared to $14.0 \pm 1.9$ events expected within the SM. Figure 4 (left) shows the distribution of the hadronic transverse momentum $p_{T}^{X}$ in the full HERA $e^{+} p$ data. The main SM production processes for events with high- $p_{T}$ multi-lepton final states are photon-photon interactions with two or all three final state leptons detected. Using the combined H1 and ZEUS data [12] with an integrated luminosity of $0.94 \mathrm{fb}^{-1}, 7$ events with $\sum p_{T}>100 \mathrm{GeV}$ were observed in the $e^{+} p$ data compared to a prediction of $1.94 \pm 0.17$ events. Figure 4 (right) shows the distribution of the scalar sum of the transverse momenta $\sum p_{T}$ for all HERA $e^{+} p$ data.

\section{Conclusions}

Recent searches for new physics in $e^{ \pm} p$ collisions performed by the $\mathrm{H} 1$ and ZEUS collaborations have been reported. In general good agreement with the SM prediction is observed for all the investigated phenomena. Limits were derived on the effective quark radius, the effective mass scale in eeqq contact interactions, the effective Planck mass scale in models with large extra dimensions, the ratio of Yukawa coupling to mass for heavy $1^{\text {st }}$ generation leptoquarks, the Yukawa couplings as a function of mass for $2^{\text {nd }} / 3^{\text {rd }}$ generation leptoquarks and squarks in R-parity violating SUSY models. Limits on the ratio of the coupling to the compositeness scale, $f / \Lambda$, as a function of the excited fermion mass were also set. In addition, searches for new physics in exclusive final states such as events with isolated leptons and large missing transverse momentum or high- $p_{T}$ multi-lepton final states were presented. 

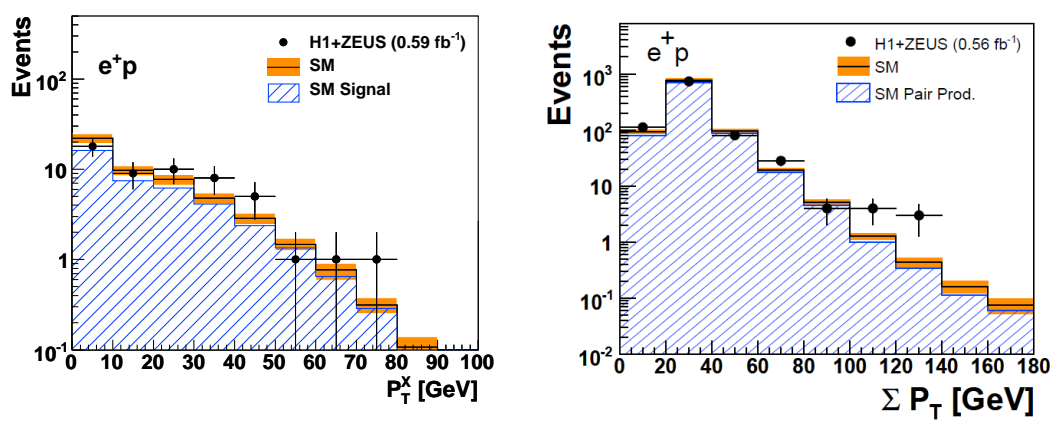

Figure 4: Distribution of the hadronic transverse momentum $p_{T}^{X}$ of events with an isolated lepton (e or $\mu$ ) and missing transverse momentum in the full HERA $e^{+} p$ data (left), and distribution of the scalar sum of the transverse momenta $\sum p_{T}$ for combined di-lepton and tri-lepton topologies for all HERA $e^{+} p$ data (right).

\section{References}

[1] H1 Collaboration, H1prelim-10-161.

[2] ZEUS Collaboration, EPS 09 contributed paper, abstract 254, ZEUS-prel-09-013.

[3] N. Arkani-Hamed, S. Dimopoulos and G. R. Dvali, Phys. Lett. B 429 (1998) 263.

[4] W. Buchmüller, R. Rückl and D. Wyler, Phys. Lett. B 191 (1987) 442 [Erratum-ibid. B 448 (1999) 320].

[5] H1 Collaboration, H1prelim-10-061.

[6] H1 Collaboration, H1prelim-10-063.

[7] B. Abbott et al. [D0 Collaboration], Phys. Rev. Lett. 83 (1999) 4476.

[8] H. K. Dreiner, arXiv:hep-ph/9707435.

P. Langacker, Phys. Lett. B 256 (1991) 277.

[9] A. Aktas et al. [H1 Collaboration], Eur. Phys. J. C 36 (2004) 425.

[10] F. D. Aaron et al. [H1 Collaboration], Phys. Lett. B 663 (2008) 382.

F. D. Aaron et al. [H1 Collaboration], Phys. Lett. B 666 (2008) 131.

F. D. Aaron et al. [H1 Collaboration], Phys. Lett. B 678 (2009) 335.

[11] F. D. Aaron et al. [H1 and ZEUS Collaboration], JHEP 1003 (2010) 035.

[12] F. D. Aaron et al. [H1 and ZEUS Collaboration], JHEP 0910 (2009) 013. 